\title{
Extracorporeal Shock Wave Therapy (ESWT) for Refractory Fractures at the Fifth Metatarsal Base
}

\section{Kenta Tanaka ${ }^{1,2,3^{*}}$, Akihiro Kanamori ${ }^{1,3}$, Masaya Kajiwara ${ }^{1}$, Tomofumi Nishino ${ }^{1}$, Yusuke Nishida $^{1}$ and Masashi Yamazaki ${ }^{1,3}$}

${ }^{1}$ Department of Orthopaedic Surgery, Faculty of Medicine, University of Tsukuba, Japan

${ }^{2}$ Department of Orthopaedic Surgery, Hitachi Ltd, Hitachinaka General Hospital, Japan

${ }^{3} T$ sukuba sports medicine and health science center

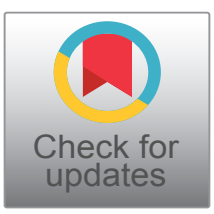

*Corresponding author: Kenta Tanaka, Department of Orthopaedic Surgery, Faculty of Medicine, University of Tsukuba, Hitachinaka General Hospital, Japan, Tel: +81298533219 , Fax: +81298533162

\begin{abstract}
Background: A fifth metatarsal base fracture is common in athletes and is often difficult to treat. Refractory cases are often managed surgically with screw fixation or bone grafts, but such procedures are extremely invasive. Recently, positive results have been reported from the use of extracorporeal shock wave therapy (ESWT) for stress fractures or delayed fracture union. We report on our experience with ESWT used in five cases of refractory fifth metatarsal base fracture where bone union had not been achieved at 12 weeks after initial treatment.

Methods: The patients included three males and two females, with a mean age of 19.2-years. The athletes were engaged in the following sporting disciplines: soccer $(n=$ 2 ), basketball ( $n=2$ ), and track and field (long jump; $n=1$ ). Mean duration of symptom before ESWT was 7.4 months. ESWT (Epos Ultra ${ }^{\circledR}$, manufactured by Dornier MedTech) was performed 3500 shocks per round of treatment were applied at an energy density of $0.36 \mathrm{~mJ} / \mathrm{mm}^{2}$ (total 1300 $\mathrm{mJ}$ ), with the shocks targeted at the point of most intense pain.
\end{abstract}

Results: Bone union was achieved in all five patients. Mean time to bone union was 11.2 weeks (7-16 weeks). Refracture occurred in one patient, but ultimately bone union was achieved with additional ESWT and the patient was able to resume sporting activities.

Conclusion: ESWT appears to be a safe and effective treatment option for refractory fifth metatarsal base fracture.

\section{Keywords}

Fifth metatarsal base fracture, Shockwave therapy

\section{Introduction}

A fifth metatarsal base fracture (Jones fracture) is a fracture located $15-20 \mathrm{~mm}$ distally from the tuberosity of the fifth metatarsal. This type of fracture, first described by Jones in 1902 [1,2] is common in athletes and is often difficult to treat $[3,4]$, with patients experiencing delayed healing, non-union, and refracture.

Refractory cases are often managed surgically with screw fixation or bone grafts $[5,6]$, but such procedures are extremely invasive. Recently, positive results have been reported from the use of extracorporeal shock wave therapy (ESWT) for stress fractures or delayed fracture union [7-10]. Compared with surgical methods, ESWT produces fewer complications with the same bone union rates [7-10] and is now viewed as a new treatment option for refractory stress fractures.

Here we add to the literature with a report on our experience with ESWT used in five cases of refractory Jones fractures where bone union had not been achieved at 12 weeks after initial treatment.

\section{Patients}

We report on five athletes who sustained a Jones fracture in one foot between December 2013 and August 2015. The patients were treated at other hospitals with either surgery or conservative therapy but did not achieve bone union. In this report, the athlete did not achieve bone union more than 12-weeks 
Table 1: Subject and their pretreatment.

\begin{tabular}{|l|l|l|l|l|l|l|l|}
\hline Sub & Sex & age & Event & Mechanism of injury & $\begin{array}{l}\text { Duration of symptoms } \\
\text { before ESWT (months) }\end{array}$ & $\begin{array}{l}\text { Operation } \\
\text { before } \\
\text { ESWT }\end{array}$ & $\begin{array}{l}\text { LIPUS before } \\
\text { ESWT }\end{array}$ \\
\hline $\mathbf{1}$ & F & 20 & Long jump & Gradually developing & 3 & - & - \\
\hline $\mathbf{2}$ & M & 20 & Basketball & Gradually developing & 3 & - & + \\
\hline & & & & $\begin{array}{l}\text { Acute } \\
\text { Non contact } \\
\text { (refracture) }\end{array}$ & 3 & - & + \\
\hline $\mathbf{3}$ & M & 19 & Soccer & Gradually developing & 14 & & \\
\hline $\mathbf{4}$ & F & 20 & Basketball & Sub acute & 11 & - & + \\
\hline $\mathbf{5}$ & M & 17 & Soccer & Gradually developing & 3 & + & + \\
\hline
\end{tabular}

Table 2: The treatment and progress.

\begin{tabular}{|l|l|l|l|l|l|}
\hline Sub & Sex & Event & Number of rounds & $\begin{array}{l}\text { The time to union } \\
\text { (weeks) }\end{array}$ & $\begin{array}{l}\text { The time to return to } \\
\text { sports (weeks) }\end{array}$ \\
\hline $\mathbf{1}$ & F & Long jump & 2 & 9 & 12 \\
\hline $\mathbf{2}$ & M & Basketball & 1 & 4 & 4 \\
\hline & & & 2 (after refracture) & 9 & 12 \\
\hline $\mathbf{3}$ & M & Soccer & 2 & 14 & 19 \\
\hline $\mathbf{4}$ & F & Basketball & 3 & 16 & 24 \\
\hline $\mathbf{5}$ & M & Soccer & 1 & 8 & 12 \\
\hline
\end{tabular}

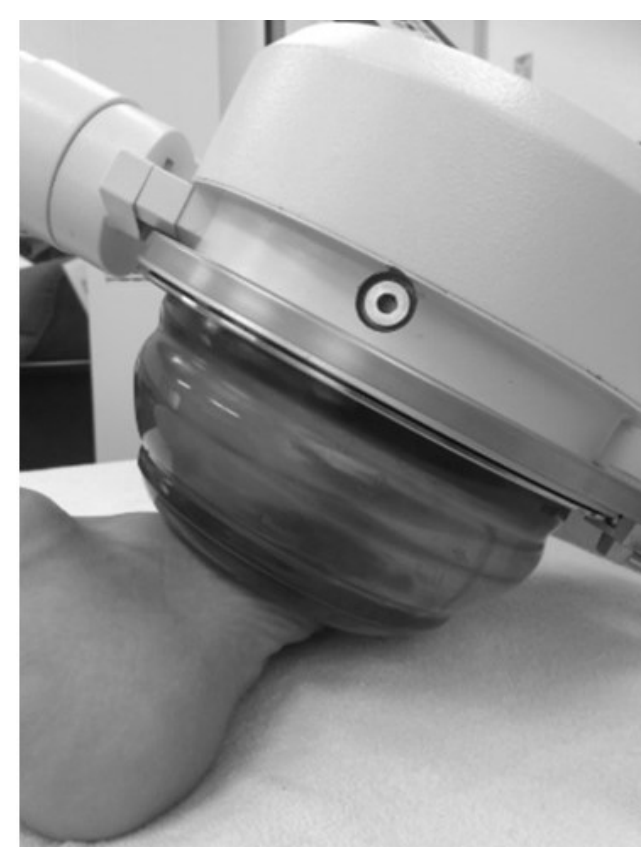

Figure 1: Appearance of ESWT for Jones fracture.

after the original injury had occurred are included. All patients sustained a Jones fracture by overuse. The patients included three males and two females, with a mean age of 19.2-years. The athletes were engaged in the following sporting disciplines: soccer $(n=2)$, basketball $(n=2)$, and track and field (long jump; $n=$ 1). Initial treatments were surgery (screw fixation; $n=$ 2 ) or conservative therapy; stop each sport activity ( $n=$ 3). LIPUS had been used before ESWT in 3 cases. Mean duration of symptom before ESWT was 7.4-months (Table 1).

\section{Methods}

ESWT (Epos Ultra ${ }^{\circledR}$, manufactured by Dornier MedTech) was performed once every 4 weeks, with between one and three rounds of treatment until signs of bone union were observed. It was defined bone union as the bone fracture line approved unclear by X-ray. 3500 shocks per round of treatment were applied at an energy density of $0.36 \mathrm{~mJ} / \mathrm{mm}^{2}$ (total $1300 \mathrm{~mJ}$ ), with the shocks targeted at the point of most intense pain, after imaging and ultrasound confirmation of the site (Figure 1). Care giving doctor held patient's leg by hand during ESWT. All patients did not use LIPUS together with shock wave. Mean follow-up time was 11-months.

\section{Results}

Bone union was achieved in all five patients. Mean time to bone union was 11.2-weeks (7-16 weeks) and mean time from first round of ESWT to return to sports activity was 15.8-weeks (12-24 weeks) (Table 2). Refracture occurred in one patient, but ultimately bone union was achieved with additional ESWT and the patient was able to resume sporting activities. Another patient retired from their sporting discipline during the treatment period, but bone union was subsequently achieved after retirement and the patient was able to continue their sport at a recreational level.

Patient 1: 20-year-old female, long jump athlete: In June 2015, pain around the fifth metatarsal gradually worsened, preventing the patient from training. The patient was diagnosed with a stress fracture of the fifth metatarsal at another hospital and conservative 


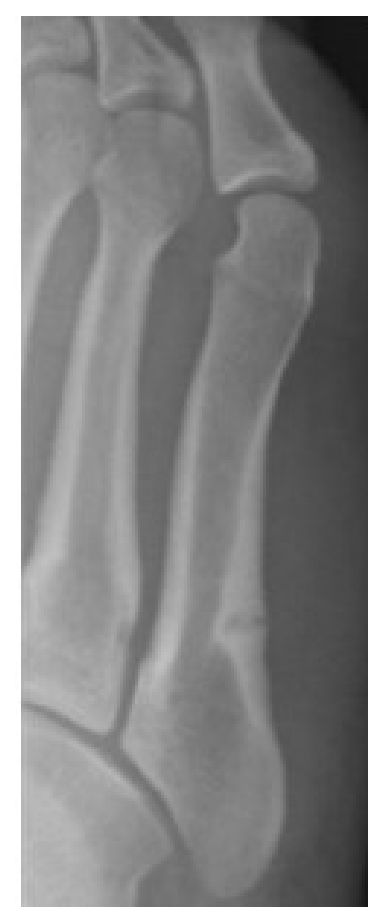

Figure 2a: X-ray at the time of injury in Patient 1.

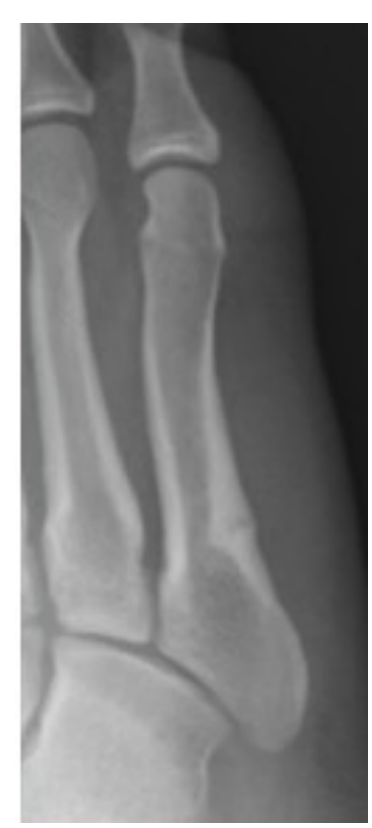

Figure 2b: X-ray at the time of pre ESWT in Patient 1.

therapy was chosen because the fracture line was restricted to the cortical bone (Figure 2a). However, bone union had not been achieved at 3 months after the original injury, so the patient attended a consultation at our hospital. The patient was treated with two rounds of ESWT over a period of 4 weeks and bone union was achieved at 9 weeks after the first round of treatment (Figure $2 \mathrm{~b}$ ). The patient returned to competition after 12 weeks (Figure 2c).

Patient 2: 20-year-male, basketball player: In February 2013, pain around the fifth metatarsal gradually worsened. The patient was diagnosed with a stress fracture of the fifth metatarsal (Figure 3a).

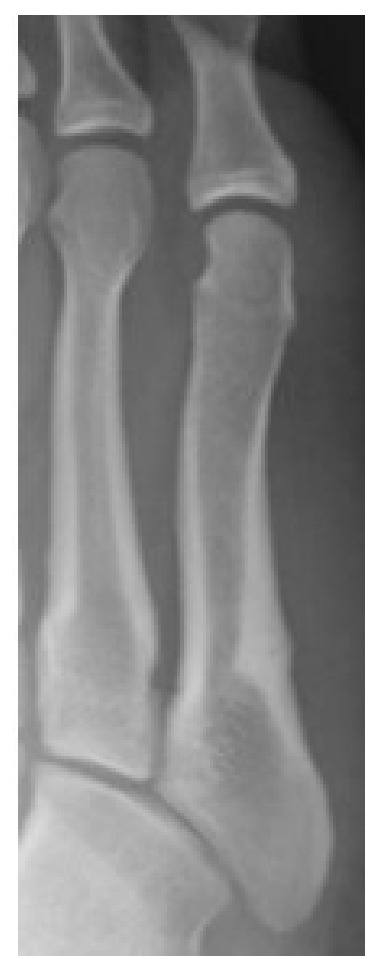

Figure 2c: X-ray at 12 weeks after ESWT in Patient 1.

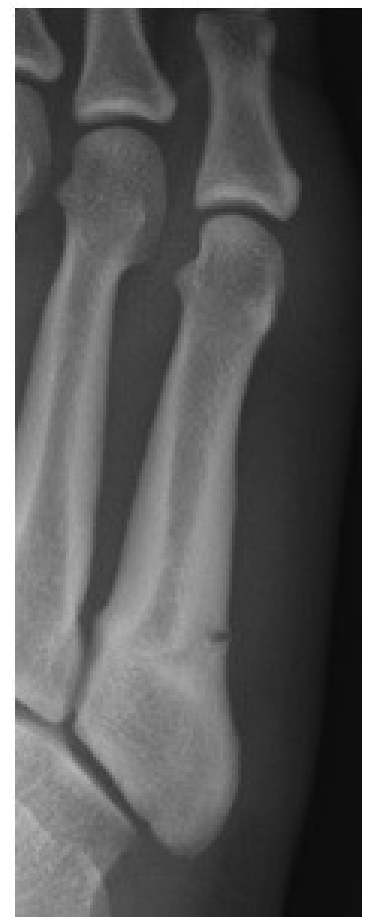

Figure 3a: X-ray at 10 months after conservative therapy in Patient 2.

However, bone union had not been achieved at 3 months after the original injury, so the patient was treated with one round of ESWT and fracture line became unclear at 4-weeks after the first round of treatment (Figure $3 \mathrm{~b}$ ). And he returned to basketball because of pain relief. However, he got pain around the fifth metatarsal again and refracture occurred at 3 months after ESWT (Figure 3c). The patient received surgery, screw fixation. However, bone union had not 


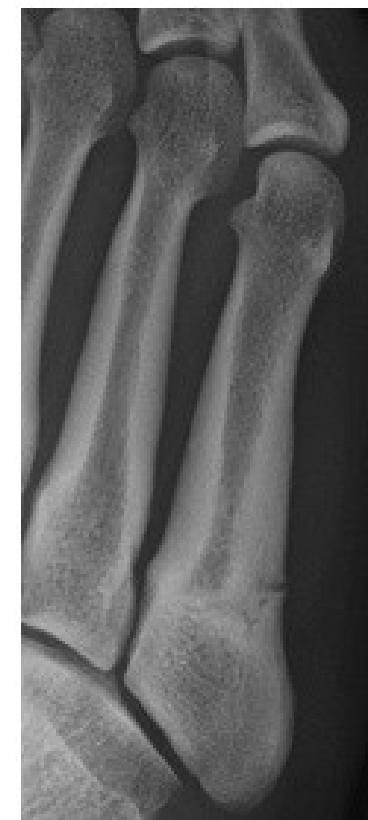

Figure 3b: X-ray at 4 weeks after ESWT in Patient 2.

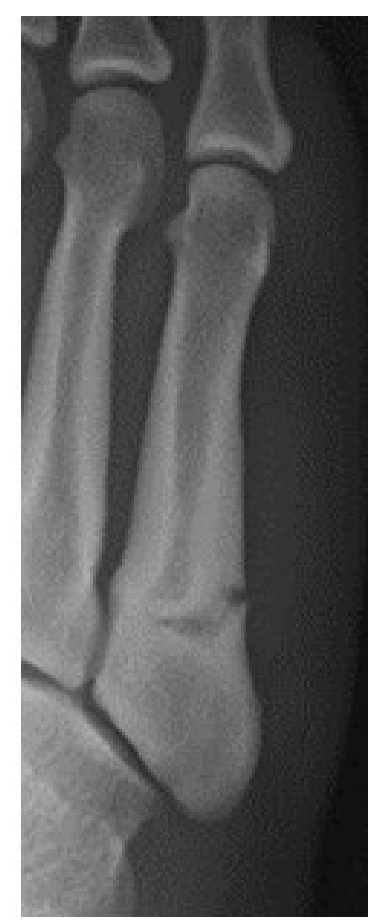

Figure 3c: X-ray of refracture at 3 months after ESWT in Patient 2.

been achieved at 11-weeks after the refracture (Figure $3 d)$. And then he was treated with two rounds of ESWT again over a period of 4-weeks and bone union was achieved at 9-weeks after the first round of re-ESWT (Figure 3e). He returned to competition at 12-weeks after ESWT.

Patient 3: 19-year-old male, soccer player: In August 2015, pain around the fifth metatarsal gradually worsened, preventing the patient from training. The patient had been diagnosed with a stress fracture of the fifth metatarsal in July 2014, and it was non union. He

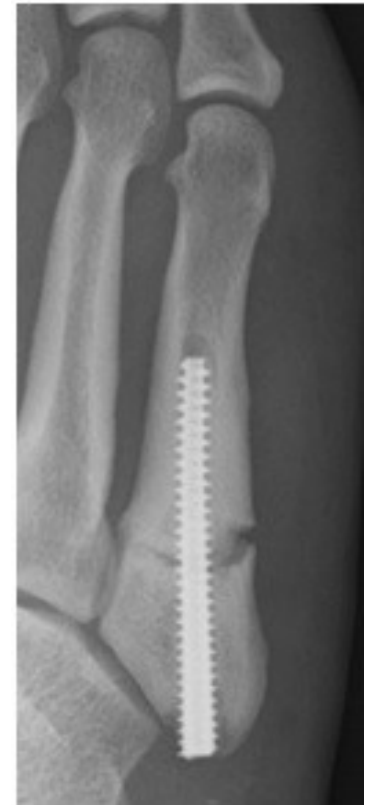

Figure 3d: X-ray of nonunion at 11 weeks after surgery in Patient 2.

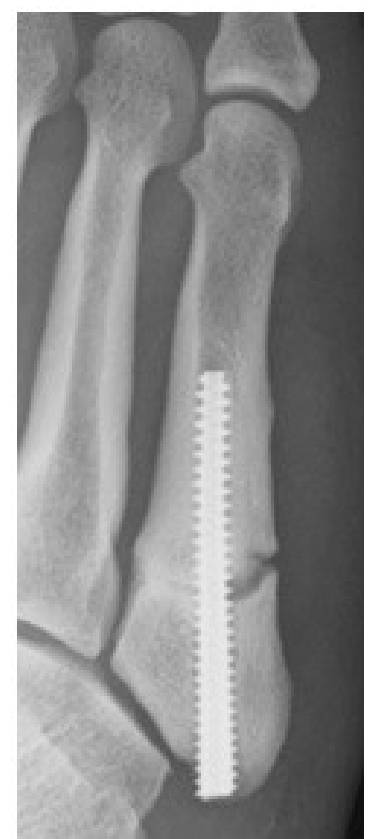

Figure 3e: X-ray of at 9 weeks after re-ESWT in Patient 2.

had put up with the pain and kept playing soccer until that time. However, bone union had not been achieved and had not relief the pain at 14-months after the original injury (Figure 4a), so he was treated with two rounds of ESWT. Bone union was achieved at 14-weeks after the treatment and pain was relieved (Figure 4b). The patient returned to competition 19 weeks after the first round of ESWT.

Patient 4: 20-year-old female, basketball player: In November 2014, pain around the fifth metatarsal after training, preventing the patient from running. The patient was diagnosed with a subacute stress fracture of the fifth metatarsal by his team doctor and received 


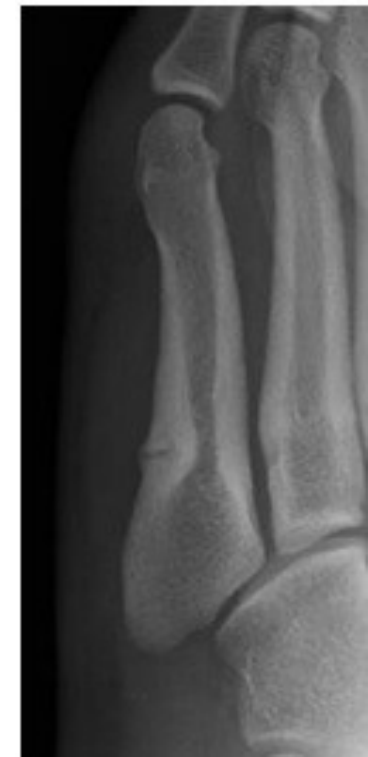

Figure 4a: X-ray at the time of pre ESWT in Patient 3.

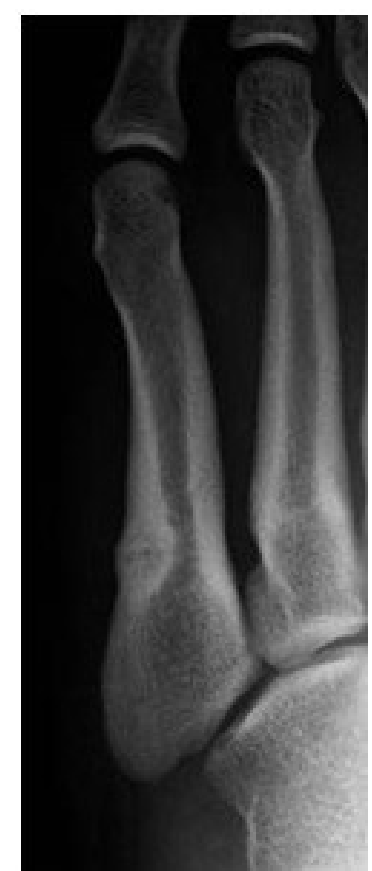

Figure 4b: X-ray at 14 weeks after ESWT in Patient 3.

surgery of screw fixation. However, bone union had not been achieved and had not relief the pain at 11-months after surgery, so the patient attended a consultation at our hospital. The patient was treated with three rounds of ESWT and bone union was achieved at 16 weeks after the first round of ESWT. The patient could not return to competition, and then return to recreational level basketball 24-weeks after the first round of ESWT.

Patient 5: 17-year-old male, soccer player: In February 2015, pain around the fifth metatarsal gradually worsened, preventing the patient from best performance. The patient was diagnosed with a stress fracture of the fifth metatarsal by his team doctor and conservative therapy was chosen because the fracture line was restricted to the cortical bone. However, bone union had not been achieved and had not relief the pain at 3-months after the original injury, so the patient attended a consultation at our hospital. The patient was treated with one round of ESWT, because he had been absent from the second round, and bone union was achieved at 8-weeks after the treatment. The patient returned to competition 12-weeks after the first round of ESWT.

\section{Discussion}

Jones fractures are commonly reported in athletes [5] and all the patients described in this paper were athletes. Intramedullary screw fixation has been reported to produce better treatment outcomes than conservative therapy in athletes [11], but conservative therapy can be chosen if the fracture line is restricted to the cortical bone. It is fairly common for cases to prove difficult to treat with both these treatment methods, with the patient experiencing delayed healing, nonunion, or refracture $[3,4]$.

A number of papers have reported that refractory cases of fracture non-union are often treated with surgery, where a clean break is made at the non-union site before bone transplantation or screw fixation [5]. These are definitely not minimally invasive procedures for athletes.

ESWT has been used as a standard modality in the field of urology for around 40-years. Orthopedic surgeons started applying this method following the chance observation that shock waves for lithotripsy resulted in increased bone density in the pelvis. In 1991, Valchanov and Michailov were the first to report positive outcomes with the use of ESWT for fracture non-union [10]. There have been numerous reports since then on the use of ESWT at fracture non-union sites, both in basic research and clinical fields $[7,8,10]$.

In terms of the mechanism by which ESWT promotes bone union at the fracture site, one study reported that shockwave causes microfractures and microbleeds at the fracture site, which induces new bone formation by promoting osteoblast production and angiogenesis [12]. Another study reported shock wave stimulates ossification of mesenchymal cells [13].

In our study as well, X-ray imaging clearly showed promotion of bone union after ESWT and we think this occurred by a mechanism similar to that reported in previous research.

Furia, et al. reported on a comparison of ESWT and surgical methods to treat non-union: The research compared outcomes in 43 patients with difficult-totreat Jones fractures treated with either ESWT ( $\mathrm{n}=$ 23) or screw fixation $(n=20)$ [9]. The results showed no difference in bone union rates between the two treatment groups, but complications in only one patient in the ESWT group versus 11 patients in the surgery 
group. The authors concluded that ESWT was a safe and effective method for refractory Jones fractures.

In our study, all five patients achieved bone union and no complications were observed. A refracture occurred in one patient after returning to competition, but bone union was achieved after the additional ESWT, and this patient is now competing again. It is inferable the refracture in this case was caused by early returning to play basketball. Protocol of after treatment was changed after this case. Patient should spend enough time, at least 12 weeks, to return their sports activity. Another patient retired from their sport during the treatment, but bone union was achieved after the retirement was announced and the patient is now participating in their sport at a recreational level. It can be described that ESWT is a safe and effective method for refractory Jones fractures.

On the other hand, ESWT has a problem. The best protocol of ESWT for fracture non-union, number of rounds of treatment, and intervals between treatment, and energy flux density is unknown. Several researchers treat their own protocol. Of course, their results are excellent across the board, but protocols are not same. When the best protocol of ESWT is revealed, union rate will be better.

As a limitation of this report, there are few patients who have Jones fractures therefore difficult to do RCT.

\section{Conclusion}

ESWT was used for refractory Jones fractures and bone union was achieved in all five patients. ESWT appears to be a safe and effective treatment option for refractory Jones fractures.

\section{COI}

None.

\section{Disclosure of Funding}

None.

\section{References}

1. Jones $R$ (1902) Fracture of the base of the fifth metatarsal bone by indirect violence. Ann Surg 35: 697-702.

2. Logan AJ, H Dabkea, D Finlayb, N Makwanaa (2007) Fifth metatarsal base fractures: A simple classification. Foot Ankle Surg 13: 30-34.

3. Larson CM, Almekinders LC, Taft TN, Garrett WE (2002) Intramedullary screw fixation of jones fractures. Analysis of failure. Am J Sports Med 30: 55-60.

4. Zelko RR, Torg JS, Rachun A (1979) Proximal diaphyseal fractures of the fifth metatarsal-treatment of the fractures and their complications in athletes. Am J Sports Med 7: 95101.

5. Hunt KJ, Anderson RB (2011) Treatment of jones fracture nonunions and refractures in the elite athlete: Outcomes of intramedullary screw fixation with bone grafting. Am J Sports Med 39: 1948-1954.

6. Rick W Wright, David A Fischer, Robert A Shively, Heidt RS Jr, Nuber GW (2000) Refracture of proximal fifth metatarsal (Jones) fracture after intramedullary screw fixation in athletes. Am J Sports Med 28: 732-736.

7. Alkhawashki HM (2015) Shock wave therapy of fracture nonunion. Injury 46: 2248-2252.

8. Schaden W, Mittermayr R, Haffner N, Smolen D, Gerdesmeyer L, et al. (2015) Extracorporeal shockwave therapy (ESWT)-First choice treatment of fracture nonunions? Int J Surg 24: 179-183.

9. John P Furia, Paul J Juliano, Allison M Wade, Schaden W, Mittermayr R (2010) Shock wave therapy compared with intramedullary screw fixation for nonunion of proximal fifth metatarsal metaphyseal-diaphyseal fractures. J Bone Joint Surg Am 92: 846-854.

10. Valchanou VD, Michailov P (1991) High energy shock waves in the treatment of delayed and nonunion of fractures. Int Orthop 15: 181-184.

11. Mologne TS, Lundeen JM, Clapper MF, O'Brien TJ (2000) Early screw fixation versus casting in the treatment of acute jones fractures. Am J Sports Med 33: 970-975.

12. Wang CJ, Wang FS, Huang CC, Yang KD, Weng LH, et al. (2005) Treatment for osteonecrosis of the femoral head: Comparison of extracorporeal shock waves with core decompression and bone-grafting. J Bone Joint Surg 87: 2380-2387.

13. Wang FS, Yang KD, Chen RF, Wang CJ, Sheen Chen SM (2002) Extracorporeal shock wave promotes growth and differentiation of bone-marrow stromal cells towards osteoprogenitors associated with induction of TGF-beta1. J Bone Joint Surg Br 84: 457-461. 